\title{
Non-perturbative Heavy Quark Effective Theory: An application to semi-leptonic B-decays
}

\author{
Michele Della Morte ${ }^{\mathrm{a}, \mathrm{b}}$, Jochen Heitger ${ }^{\mathrm{c}}$, Hubert Simma $^{\mathrm{d}}$, Rainer Sommer ${ }^{\mathrm{d}, \mathrm{e}, *}$ \\ ${ }^{a}$ CP3-Origins $\mathcal{E}$ Danish IAS, University of Southern Denmark, Campusvej 55, DK-5230 Odense M, Denmark \\ ${ }^{b}$ IFIC (CSIC), c/ Catedrático José Beltrán, 2. E-46980, Paterna, Spain \\ ${ }^{c}$ Westfälische Wilhelms-Universität Münster, Institut für Theoretische Physik, Wilhelm-Klemm-Straße 9, D-48149 Münster, Germany \\ ${ }^{d}$ John von Neumann Institute for Computing (NIC), DESY, Platanenallee 6, 15738 Zeuthen, Germany \\ ${ }^{e}$ Institut für Physik, Humboldt Universität, Newtonstr. 15, 12489 Berlin, Germany
}

\begin{abstract}
We review a lattice strategy how to non-perturbatively determine the coefficients in the HQET expansion of all components of the heavy-light axial and vector currents, including $1 / m_{\mathrm{h}}$-corrections. We also discuss recent preliminary results on the form factors parameterizing semi-leptonic B decays at the leading order in $1 / m_{\mathrm{h}}$.
\end{abstract}

Keywords: Lattice QCD, Heavy Quark Effective Theory, Flavour Physics, Bottom quarks, Meson decay

\section{Introduction}

B-meson decays present an excellent opportunity to test the Standard Model and to search for New Physics. Many decay channels are allowed. Their rates depend on CKM matrix elements and often have a sensitivity to heavy particles both within and outside the Standard Model. Such effects provide us with indirect tests of New Physics which are complementary to direct searches for new particles at very high energy. In this way, B-decays provide for stringent constraints on the structure and couplings of theories beyond the Standard Model. Of course, to make this program work, the theory of these decays needs to be understood. Here the challenge is in the correct handling of QCD.

The relevant intrinsic QCD scale is much smaller than the mass scale of the electroweak interactions or any New Physics. Therefore, the effect of beyond the Standard Model interactions can accurately be described by a perturbatively treated effective Hamiltonian, consisting e.g. of bilinear quark currents or local four-quark

\footnotetext{
${ }^{*}$ Corresponding author

Email address: rainer . sommer@desy . de (Rainer Sommer)
}

interactions. One then has to compute the hadronic matrix elements of this effective Hamiltonian with the basic theory given just by QCD (with $N_{\mathrm{f}}=5$ flavours).

A first principles evaluation of the matrix elements is possible by lattice QCD, non-perturbatively in the QCD coupling but, as mentioned, perturbatively in the effective Hamiltonian, which has contributions from the electroweak sector and, possibly, from New Physics. We do, however, still have to treat a hierarchy of scales

$$
L^{-1} \ll m_{\pi} \approx 140 \mathrm{MeV} \ll m_{\mathrm{B}} \approx 5 \mathrm{GeV} \ll a^{-1} .
$$

This forbids direct simulations on a lattice with spatial extent $L$ and lattice spacing $a$ on nowadays computers. We therefore apply another effective theory, HQET, which provides us (at appropriate kinematics) with the expansion of QCD observables in inverse powers of $m_{\mathrm{h}}$. The mass scale in the numerator of this expansion parameter is the intrinsic QCD-scale of around $500 \mathrm{MeV}$ as well as (in the rest frame of the B-meson) small spatial momenta. This theory, put on the lattice, only has to cover accurately the scales far below $m_{\mathrm{B}}$.

HQET has low-energy constants (or HQET parameters) which need to and can be determined nonperturbatively, as explained in [1]. Besides the HQET 
parameters in the action, there are a number of parameters for each considered composite field which makes up the electroweak Hamiltonian. Extending the procedure discussed in [1] for the parameters in the action and the temporal component of the axial current, we review here a matching strategy for the full set of temporal and spatial components of the heavy-light vector and axial currents.

These are of relevance for the determination of the CKM elements. For instance, $V_{\mathrm{ub}}$, can be determined from the inclusive decays $\mathrm{B} \rightarrow X_{\mathrm{u}} \ell v$ [2, sect. 12]

$$
\left|V_{\mathrm{ub}}\right|=\left(4.41 \pm 0.15_{-0.19}^{+0.15}\right) \times 10^{-3},
$$

or from various exclusive decays. From the exclusive semi-leptonic decay $\mathrm{B} \rightarrow \pi \ell v$, one finds [2, sect. 12]

$$
\left|V_{\mathrm{ub}}\right|=(3.28 \pm 0.29) \times 10^{-3},
$$

while the purely leptonic channel, which is more difficult in experiment, tends to give values of $\left|V_{\mathrm{ub}}\right|$ that are significantly larger than in eq. (2). For instance, using the branching ratio $\mathcal{B}(\mathrm{B} \rightarrow \tau v)=(1.14 \pm 0.22) \times 10^{-4}$ one obtains [2, sect. 12]

$$
\left|V_{\mathrm{ub}}\right|=(4.22 \pm 0.42) \times 10^{-3} .
$$

The discrepancies between these different determinations of $\left|V_{\mathrm{ub}}\right|$ are not understood. We need both theoreti$\mathrm{cal}$ and experimental improvements to find out their origin. For exclusive decays, such as $\mathrm{B} \rightarrow \tau v$ or $\mathrm{B} \rightarrow \pi \ell v$, it is known how to compute the required hadronic matrix elements by lattice computations. In this respect, the purely leptonic decay channel $\mathrm{B} \rightarrow \tau v$, is the simplest case and the required decay constant $f_{\mathrm{B}}$ is known precisely. We refer to [3] and references therein for a summay.

Semi-leptonic decays, such as $\mathrm{B} \rightarrow \pi \ell v$ or $\mathrm{B}_{s} \rightarrow \mathrm{K} \ell v$ allow to extract the CKM parameter $\left|V_{\mathrm{ub}}\right|$ from a combination of the experimental differential decay rate with a theoretical determination of the form factor $f_{+}\left(q^{2}\right)$. In particular lattice QCD can achieve the computation of $f_{+}\left(q^{2}\right)$. In principle, it is sufficient to do this at a single value of $q^{2}$, the squared momentum transfer. However, in practice, experimental data is provided over a range (of bins) of $q^{2}$, and one has to use some knowledge on the functional form of $f_{+}\left(q^{2}\right)$, e.g. in the form of the BCL paramterisation [4]. Then, a theoretical prediction of $f_{+}\left(q^{2}\right)$ for a single $q^{2}$ is sufficient to extract $\left|V_{\mathrm{ub}}\right|$. In section 4 , we investigate the feasibility of a precise lattice determination of the form factor in the continuum limit and at a fixed $q^{2}$. Of course, eventually the precision will be enhanced when the form factor is known at more than one $q^{2}$.

\section{General matching strategy}

Following $[5,6,7,8]$, we fix the 19 parameters in the HQET expansion of the action and vector/axial currents through matching equations, which we write in the form

$$
\Phi_{i}^{\mathrm{QCD}}\left(L, m_{\mathrm{h}}, 0\right)=\Phi_{i}^{\mathrm{HQET}}\left(L, m_{\mathrm{h}}, a\right) .
$$

In the above equations the $\Phi_{i}(i=1 \ldots 19)$ are finitevolume, renormalized, QCD quantities defined in the continuum

$$
\Phi_{i}^{\mathrm{QCD}}\left(L, m_{\mathrm{h}}, 0\right)=\lim _{a \rightarrow 0} \Phi_{i}^{\mathrm{QCD}}\left(L, m_{\mathrm{h}}, a\right)
$$

whereas the $\Phi_{i}^{\mathrm{HQET}}$ are understood to be expanded up to a given order in $1 / m_{\mathrm{h}}$ (NLO in our setup) and computed in HQET at a finite lattice spacing. The $1 / m_{\mathrm{h}}$-expansion of the observables $\Phi_{i}$ results from expanding the action and composite fields $O^{\mathrm{QCD}}(x)$, viz.,

$$
O^{\mathrm{HQET}}\left(m_{\mathrm{h}}\right)=Z_{O}\left\{O^{\mathrm{stat}}+\sum_{n} c_{n} O_{n}\right\} .
$$

The coefficients in the expansion are chosen such that eq. (4) holds. In the r.h.s. of the above equation one has to include all linearly independent operators with mass dimension one higher than $O^{\mathrm{QCD}}$ (or $O^{\text {stat}}$ ) transforming in the same way as $O^{\mathrm{QCD}}$ under the common set of symmetries of QCD and HQET. These are needed for the renormalization and $\mathrm{O}(a)$ improvement of the effective theory, and in order to account in HQET for the $m_{\mathrm{h}}$ effects of QCD. Such effects are described by the $m_{\mathrm{h}}$ dependence of the parameters $Z_{O}$ and $c_{n}$ in eq. (6). Once the matching is performed at a given value of the heavy quark mass and fixed $L=L_{1}$, the $L$-dependence in the difference $\Phi_{i}^{\mathrm{QCD}}\left(L, m_{\mathrm{h}}, 0\right)-\Phi_{i}^{\mathrm{HQET}}\left(L, m_{\mathrm{h}}, a\right)$ quantifies higher-order (in $1 / m_{\mathrm{h}}$ ) corrections. These have been studied at tree-level in [8] for a particular class of matching conditions. Note that at tree-level and in the continuum limit, the $L$-dependence of dimensionless observables $\Phi_{i}$ appears only in the combination $z=L m_{\mathrm{h}}$.

\subsection{Definition of the matching observables}

Let us assume we want to determine the parameters appearing at NLO in the expansion of a current $J$.

The matching observables can be constructed from suitable combinations of correlation functions, $C_{J}$, which typically have a single insertion of $J$. Writing the HQET expansion of $J$ as

$$
J^{\mathrm{HQET}}=Z_{J}^{\mathrm{HQET}}\left\{J^{\mathrm{stat}}+\sum_{n} c_{J_{n}} J_{n}\right\}+\mathrm{O}\left(1 / m_{\mathrm{h}}^{2}\right),
$$


then the correlation functions take the generic form

$$
\begin{aligned}
& C_{J}^{\mathrm{HQET}}=Z_{J}^{\mathrm{HQET}} Z_{C}^{\mathrm{HQET}} e^{-m_{\text {bare }} x_{C}}\left\{C_{J}^{\text {stat }}+\right. \\
& \left.\omega_{\text {kin }} C_{J}^{\text {kin }}+\omega_{\text {spin }} C_{J}^{\text {spin }}+\sum_{n} c_{J_{n}} C_{J_{n}}^{1 / \mathrm{m}}\right\},
\end{aligned}
$$

where all correlators on the r.h.s. are computed in the static approximation. The only appearance of the bare HQET quark mass, $m_{\text {bare }}$, is in the factor $e^{-m_{\text {bare }} x_{C}}$, with $x_{C}$ given by the time distances of the heavy (static) quark fields entering in $C_{J}^{\mathrm{HQET}}$. Apart from $Z_{J}^{\mathrm{HQET}}$, all other (re)normalization factors contributing to $C_{J}^{\mathrm{HQET}}$ are collected in $Z_{C}^{\mathrm{HQET}}$.

In the correlation functions on the r.h.s. of eq. (8), the leading-order term $C_{J}^{\text {stat }}$ has just one insertion of $J^{\text {stat }}$ (instead of $J$ ), while $C_{J}^{\text {kin/spin }}$ differ from $C_{J}^{\text {stat }}$ by an extra insertion (summed over the entire space-time volume) of the $1 / m_{\mathrm{h}}$-terms $O_{\text {kin }}$ or $O_{\text {spin }}$ from the Lagrangian. The other next-to-leading contributions $C_{J_{n}}^{1 / \mathrm{m}}$ have an insertion of one of the higher-dimensional operators $J_{n}$ from the expansion in eq. (7).

The observables $\Phi_{i}$ are defined as combinations of such correlators, typically logarithms of ratios, and only one of the observables, say $\Phi_{1}$, is left with an explicit dependence on $m_{\text {bare }}$.

By combining all HQET parameters into a vector

$$
\omega=\left(m_{\text {bare }}, \omega_{\text {kin }}, \omega_{\text {spin }}, c_{J_{1}}, \ldots, \ln Z_{J}^{\mathrm{HQET}}, \ldots\right)^{T},
$$

the HQET expansion of the observables can be cast in the form

$$
\begin{aligned}
& \Phi_{i}^{\mathrm{HQET}}(L, M, a)=\eta_{i}(L, a)+ \\
& \varphi_{i}^{j}(L, a) \omega_{j}(M, a)+\mathrm{O}\left(1 / m_{\mathrm{h}}^{2}\right),
\end{aligned}
$$

where $M$ is the Renormalization Group Invariant (RGI) heavy quark mass and the vector $\eta$ represents the contribution of the static terms $C_{J}^{\text {stat }}$ in the correlators involved. In general, the existence of a continuum limit can be guaranteed only for the combination of HQET quantities which appears on the r.h.s. of eq. (10). Only at tree-level each individual term on the r.h.s. has a well defined continuum limit.

In order to learn about the structure of the matrix $\varphi_{i}^{j}$ in eq. (10), we group the HQET parameters into blocks $\left(m_{\text {bare }}\right),\left(\omega_{\text {kin }}, \omega_{\text {spin }}\right),\left(c_{J_{i}}, Z_{J}\right),\left(c_{J_{i}^{\prime}}, Z_{J^{\prime}}\right),\left(c_{J_{i}^{\prime \prime}}, Z_{J^{\prime \prime}}\right), \ldots$, and assume that $J$ is the current which is used in $\Phi_{1}$. A suitable choice of the other observables then yields the following form of the matrix $\varphi_{i}^{j}$ :

- All entries of the first column, except $\varphi_{1}^{1}$, vanish.
- In the first row, $\varphi_{1}^{2}$ and $\varphi_{1}^{3}$ are non-vanishing, due to the contributions from $\omega_{\text {kin }}$ and $\omega_{\text {spin }}$ to $\Phi_{1}$. In addition, there may be non-zero $\varphi_{1}^{j}$ with $j$ from a single block, which corresponds to the current used in $\Phi_{1}$. In our case, this comes from the $A_{0,1}$-term (i.e., a term at NLO in the HQET expansion of the temporal component of the axial current, analogous to eq. (13) below), which enters the matching condition for $m_{\text {bare }}$.

- The rest has a simple block structure, with nonzero blocks only in the second block-column (corresponding to contributions from $\omega_{\text {kin }}$ and $\left.\omega_{\text {spin }}\right)$ and in the blocks on the diagonal (corresponding to the mixings in the last term of eq. (8)).

Schematically, we have the following block structure:

$$
\varphi=\left(\begin{array}{c|c|c|c|c}
\varphi_{1}^{1} & * & * & 0 & 0 \\
\hline 0 & * & 0 & 0 & 0 \\
\hline 0 & * & * & 0 & 0 \\
\hline 0 & * & 0 & * & 0 \\
\hline 0 & * & 0 & 0 & *
\end{array}\right) .
$$

That form can be preserved when an additional (effective) operator $J^{\prime}$ is included in the matching. Therefore, the system can always be solved, and the HQET parameters can be determined, by block-wise backward substitution.

Let us explicitly discuss the case of the (renormalized) heavy-light vector current in HQET. The temporal component is

$$
V_{0}^{\mathrm{HQET}}(x)=Z_{\mathrm{V}_{0}}^{\mathrm{HQET}}\left[V_{0}^{\mathrm{stat}}(x)+\sum_{i=1}^{2} c_{\mathrm{V}_{0, i}} V_{0, i}(x)\right],
$$

with the static term

$$
V_{\mu}^{\text {stat }}(x)=\bar{\psi}_{\ell}(x) \gamma_{\mu} \psi_{\mathrm{h}}(x)
$$

and two additional dimension-four contributions

$$
\begin{aligned}
V_{0,1}(x) & =\bar{\psi}_{\ell}(x) \frac{1}{2} \gamma_{i}\left(\nabla_{i}^{\mathrm{S}}-\overleftarrow{\nabla}_{i}^{\mathrm{S}}\right) \psi_{\mathrm{h}}(x), \\
V_{0,2}(x) & =\bar{\psi}_{\ell}(x) \frac{1}{2} \gamma_{i}\left(\nabla_{i}^{\mathrm{S}}+\overleftarrow{\nabla}_{i}^{\mathrm{S}}\right) \psi_{\mathrm{h}}(x),
\end{aligned}
$$

where all derivatives are defined from symmetric nearest neighbour differences and $\psi_{\mathrm{h}}$ and $\psi_{\ell}$ denote static and light (relativistic) quark fields, respectively. In order to have access to a variety of different kinematical configurations, we will make extensive use here of generalized periodic boundary conditions for the fermions

$$
\psi(x+L \hat{k})=e^{i \theta_{k}} \psi(x), \quad \bar{\psi}(x+L \hat{k})=\bar{\psi}(x) e^{-i \theta_{k}} .
$$


The phases $\theta_{k}[9,10]$, sometimes referred to as twisting $[11,12]$, can be employed to inject a momentum $\left|\boldsymbol{\theta}_{\mathrm{h}}-\boldsymbol{\theta}_{\ell}\right| / L$ in correlation functions $[11,12]$. For example, it is clear that for $\boldsymbol{\theta}_{\mathrm{h}}=\boldsymbol{\theta}_{\ell}$ composite fields such as $V_{0,2}(x)$ above can be associated with total derivative operators and, therefore, correlation functions at zero external momenta are not sensitive to their insertions.

Considering now the spatial components of the vector current, the HQET expansions (see [13]) can be written as

$$
V_{k}^{\mathrm{HQET}}(x)=Z_{\overrightarrow{\mathrm{V}}}^{\mathrm{HQET}}\left[V_{k}^{\mathrm{stat}}(x)+\sum_{i=1}^{4} c_{\mathrm{V}_{k, i}} V_{k, i}(x)\right],
$$

with the following four extra terms:

$$
\begin{aligned}
V_{k, 1}(x) & =\bar{\psi}_{\ell}(x) \frac{1}{2}\left(\nabla_{i}^{\mathrm{S}}-\overleftarrow{\nabla}_{i}^{\mathrm{S}}\right) \gamma_{i} \gamma_{k} \psi_{\mathrm{h}}(x), \\
V_{k, 2}(x) & =\bar{\psi}_{\ell}(x) \frac{1}{2}\left(\nabla_{k}^{\mathrm{S}}-\overleftarrow{\nabla}_{k}^{\mathrm{S}}\right) \psi_{\mathrm{h}}(x), \\
V_{k, 3}(x) & =\bar{\psi}_{\ell}(x) \frac{1}{2}\left(\nabla_{i}^{\mathrm{S}}+\overleftarrow{\nabla}_{i}^{\mathrm{S}}\right) \gamma_{i} \gamma_{k} \psi_{\mathrm{h}}(x), \\
V_{k, 4}(x) & =\bar{\psi}_{\ell}(x) \frac{1}{2}\left(\nabla_{k}^{\mathrm{S}}+\overleftarrow{\nabla}_{k}^{\mathrm{S}}\right) \psi_{\mathrm{h}}(x) .
\end{aligned}
$$

The axial vector current components are listed in [8]. The classical values of the coefficients are

$$
\begin{aligned}
& c_{\mathrm{V}_{0,1}}=c_{\mathrm{V}_{0,2}}=c_{\mathrm{V}_{k, 1}}=c_{\mathrm{V}_{k, 3}}=\frac{1}{2 m_{\mathrm{h}}}, \\
& \text { and } \quad c_{\mathrm{V}_{k, 2}}=c_{\mathrm{V}_{k, 4}}=-\frac{1}{m_{\mathrm{h}}} .
\end{aligned}
$$

Concretely, as in $[5,6,7]$, it is advantageous to construct the matching observables using Schrödinger functional (SF) homogeneous boundary conditions at $x_{0}=0$ and $x_{0}=T[14,15,16]$. Two categories of QCD correlation functions are formed from composite fields in the bulk, $0<x_{0}<T$, and boundary quark fields $\zeta \ldots \bar{\zeta}^{\prime}$.

- Boundary-to-boundary correlators

$$
\begin{aligned}
& F_{1}\left(\boldsymbol{\theta}_{\ell}, \boldsymbol{\theta}_{\mathrm{b}}\right)= \\
& -\frac{a^{12}}{2 L^{6}} \sum_{\mathbf{u}, \mathbf{v}, \mathbf{y}, \mathbf{z}}\left\langle\bar{\zeta}_{\ell}^{\prime}(\mathbf{u}) \gamma_{5} \zeta_{\mathrm{b}}^{\prime}(\mathbf{v}) \bar{\zeta}_{\mathrm{b}}(\mathbf{y}) \gamma_{5} \zeta_{\ell}(\mathbf{z})\right\rangle, \\
& F_{1}^{\ell \ell}\left(\boldsymbol{\theta}_{\ell}, \boldsymbol{\theta}_{\ell^{\prime}}\right)= \\
& -\frac{a^{12}}{2 L^{6}} \sum_{\mathbf{u}, \mathbf{v}, \mathbf{y}, \mathbf{z}}\left\langle\bar{\zeta}_{\ell}^{\prime}(\mathbf{u}) \gamma_{5} \zeta_{\ell^{\prime}}^{\prime}(\mathbf{v}) \bar{\zeta}_{\ell^{\prime}}(\mathbf{y}) \gamma_{5} \zeta_{\ell}(\mathbf{z})\right\rangle, \\
& K_{1}\left(\boldsymbol{\theta}_{\ell}, \boldsymbol{\theta}_{\mathrm{b}}\right)= \\
& -\frac{a^{12}}{6 L^{6}} \sum_{k} \sum_{\mathbf{u}, \mathbf{v}, \mathbf{y}, \mathbf{z}}\left\langle\bar{\zeta}_{\ell}^{\prime}(\mathbf{u}) \gamma_{k} \zeta_{\mathrm{b}}^{\prime}(\mathbf{v}) \bar{\zeta}_{\mathrm{b}}(\mathbf{y}) \gamma_{k} \zeta_{\ell}(\mathbf{z})\right\rangle, \\
& F_{\mathrm{V}_{0}}\left(x_{0}, \boldsymbol{\theta}_{\ell}, \boldsymbol{\theta}_{\ell^{\prime}}, \boldsymbol{\theta}_{\mathrm{h}}\right)= \\
& -\frac{a^{15}}{2 L^{6}} \sum_{\mathbf{u}, \mathbf{v}, \mathbf{y}, \mathbf{z}, \mathbf{x}}\left\langle\bar{\zeta}_{\ell^{\prime}}^{\prime}(\mathbf{u}) \gamma_{5} \zeta_{\ell}^{\prime}(\mathbf{v})\left(V_{\mathrm{I}}\right)_{0}(x) \bar{\zeta}_{\mathrm{b}}(\mathbf{y}) \gamma_{5} \zeta_{\ell^{\prime}}(\mathbf{z})\right\rangle,
\end{aligned}
$$

- Bulk-to-boundary correlators

$$
\begin{aligned}
& k_{\mathrm{V}_{0}}\left(x_{0}, \boldsymbol{\theta}_{\ell}, \boldsymbol{\theta}_{\mathrm{b}}\right)= \\
& i \frac{a^{6}}{6} \sum_{k} \sum_{\mathbf{y}, \mathbf{z}}\left\langle\left(V_{\mathrm{I}}\right)_{0}(x) \bar{\zeta}_{\mathrm{b}}(\mathbf{y}) \gamma_{k} \zeta_{\ell}(\mathbf{z})\right\rangle, \\
& k_{\overrightarrow{\mathrm{V}}}\left(x_{0}, \boldsymbol{\theta}_{\ell}, \boldsymbol{\theta}_{\mathrm{b}}\right)= \\
&-\frac{a^{6}}{6} \sum_{k} \sum_{\mathbf{y}, \mathbf{z}}\left\langle\left(V_{\mathrm{I}}\right)_{k}(x) \bar{\zeta}_{\mathrm{b}}(\mathbf{y}) \gamma_{k} \zeta_{\ell}(\mathbf{z})\right\rangle, \\
& k_{\mathrm{V}_{1}}^{1}\left(x_{0}, \boldsymbol{\theta}_{\ell}, \boldsymbol{\theta}_{\mathrm{b}}\right)= \\
&-\frac{a^{6}}{2} \sum_{\mathbf{y}, \mathbf{z}}\left\langle\left(V_{\mathrm{I}}\right)_{1}(x) \bar{\zeta}_{\mathrm{b}}(\mathbf{y}) \gamma_{1} \zeta_{\ell}(\mathbf{z})\right\rangle,
\end{aligned}
$$

where the label $\mathrm{b}$ refers to heavy relativistic quarks of mass close to the b-quark mass, and the subscript I stands for $\mathrm{O}(a)$ improvement, as discussed in $[10,17]$ for Wilson quarks. The correlation functions are illustrated in [1], where it is also explained how they relate to quantum mechanical Hilbert space matrix elements.

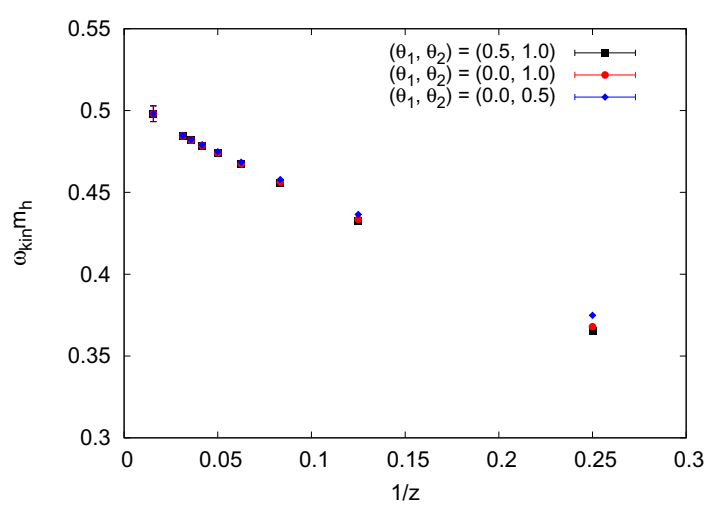

Figure 1: Tree-level continuum results for the parameter $\omega_{\text {kin }}$ in $\mathscr{L}^{\mathrm{HQET}}$.

In order to determine the parameters $\omega_{i}, 1 \leq i \leq 19$, the above correlation functions as well as some related to the axial current are combined to 19 observables [8, 18]. First of all, ratios are formed such that the renormalization of the boundary quark fields drops out. Then, one searches for combinations and particular choices of $\boldsymbol{\theta}_{i}$ such that the matrix $\varphi$ has many zeros and is easily inverted. ${ }^{1}$ Finally, one looks for choices where higher $\mathrm{O}\left(1 / m_{\mathrm{h}}^{2}\right)$ terms are rather suppressed. Such a search has successfully been carried out at tree-level of perturbation theory [8], and we discuss some of the results in the next section.

\footnotetext{
ber.
} 

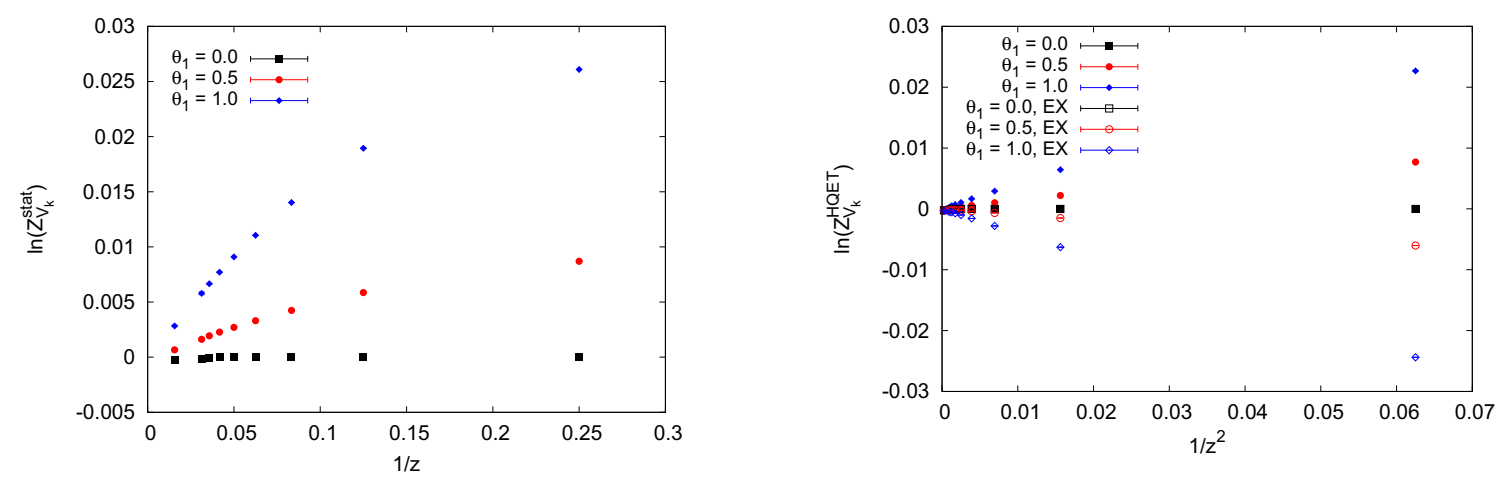

Figure 2: Tree-level continuum results for the parameter $\ln Z_{\overrightarrow{\mathrm{V}}}$ of the spatial components of the vector current, at static order versus $1 / z$ (left) and at $\mathrm{O}\left(1 / m_{\mathrm{h}}\right)$ versus $1 / z^{2}$ (right). $\boldsymbol{\theta}_{1}=0$ is clearly preferable, while already at $\boldsymbol{\theta}=(1,1,1)$ the corrections become very substantial. We also note that at tree-level and for $\boldsymbol{\theta}_{1}=0$ the dependence of the matching equations involving a single angle on any other parameter vanishes. Hence, the corresponding parameters are independent of whether or not using the "EX" setup, and higher-order contributions are absent.

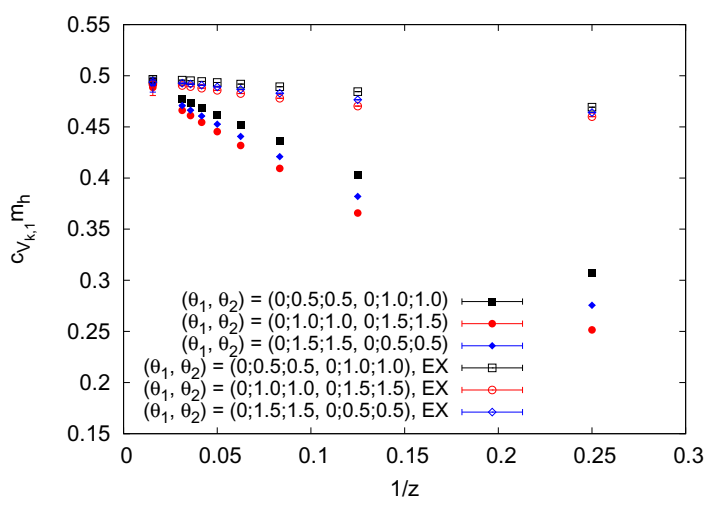

Figure 3: Tree-level continuum results for the parameter $c_{\mathrm{V}_{k, 1}}$ (depending on two angles) of the spatial components of the vector current. Smaller values of $\left(\boldsymbol{\theta}_{1}, \boldsymbol{\theta}_{2}\right)$ tend to yield smaller $1 / z$-corrections, and part of the higher-order $1 / z$-dependence (being smaller in the "EX" setup) is induced by other parameters.

\section{Tree-level study of HQET parameters}

We now illustrate a few results of the tree-level computation [8] of the HQET parameters for the vector currents that are needed for the semi-leptonic B-decays.

After choosing a suitable set of observables $\Phi_{i}$, the HQET parameters are computed from eq. (4) inserting the HQET expansion eq. (10). At tree-level, each term in eq. (10) separately has a continuum limit. Since we are here not interested in the cutoff effects, the limit is taken term by term, and we then solve the matching equations

$$
\sum_{j} \varphi_{i}^{j} \cdot \omega_{j}=\Phi_{i}^{\mathrm{QCD}}-\eta_{i}
$$

at $a=0$ for the unknown HQET parameters $\omega_{j}$. Due to the block structure of $\varphi$ and because of additional simplifications, which occur at tree-level, and with the choice of twist angles according to the strategy of [8], one can determine the parameters one after the other from the matching equations (18).

A main motivation of the tree-level study in [8] was to investigate the effect of higher-order corrections (in $\left.1 / m_{\mathrm{h}}\right)$ in the system of matching equations and to test the choice of observables.

At tree-level and in the static limit, the HQET parameters at $a=0$ must reproduce the exactly known classical values. After multiplying each parameter with the proper factors of $m_{\mathrm{h}}$, this limit is obtained for $z \equiv$ $m_{\mathrm{h}} \cdot L \rightarrow \infty$. The size of the higher-order contributions in $1 / m_{\mathrm{h}}$ can then be explored by looking at the rate with which the HQET parameters converge to their known classical values. One expects in particular

$$
\begin{aligned}
c_{\mathrm{V}_{k, 1}} m_{\mathrm{h}} & =\frac{1}{2}+\mathrm{O}(1 / z), \\
\omega_{\text {kin }} m_{\mathrm{h}} & =\frac{1}{2}+\mathrm{O}(1 / z), \\
\ln Z_{V_{0}}^{\mathrm{HQET}} & =0+\mathrm{O}(1 / z), \\
\ln Z_{V_{k}}^{\text {stat }} & =0+\mathrm{O}(1 / z), \\
\ln Z_{V_{k}}^{\mathrm{HQET}} & =0+\mathrm{O}\left(1 / z^{2}\right) .
\end{aligned}
$$

Any $\mathrm{O}(1 / z)$ deviations from the behaviour indicated in the equations above are to be understood as a sign of the $1 / m_{\mathrm{h}}$-corrections neglected in the static approximation of HQET. Once all $1 / m_{\mathrm{h}}$-contributions are included in HQET and in its matching to QCD, as assumed here throughout, also the $\mathrm{O}(1 / z)$ corrections to the HQET parameters are fixed. Consequently, deviations from a lin- 
ear $1 / z$-dependence signal higher-order corrections disregarded in the effective theory. They are also revealed by a non-vanishing dependence of the HQET parameters on the specific choices for the $\boldsymbol{\theta}$-angles on which the observables used in the matching step depend.

Within our tree-level study, where the HQET parameters can be determined one after the other from eq. (18), one can turn off the effects of $\mathrm{O}\left(1 / m_{\mathrm{h}}\right)$ corrections in the previous steps by replacing all parameters determined in previous steps by their exact classical values. One then sees directly and only the effects of the new observables considered in the present block. This procedure is dubbed "EX". We note that the difference between "EX" and the solution of the full system is dominated by the $1 / m_{\mathrm{h}}$-effects in $\omega_{\text {kin }}$ (figure 1 ).

Figure 1 shows that $\omega_{\text {kin }}$ has rather linear $1 / z$ corrections, which have little dependence on $\boldsymbol{\theta}$. The coefficient of $1 / z$ is around one, the standard magnitude which is to be expected. However, for the investigated set of $\Phi_{i}$, the other HQET parameters show much smaller corrections, as we see below for a few examples and as is discussed in more detail in [8].

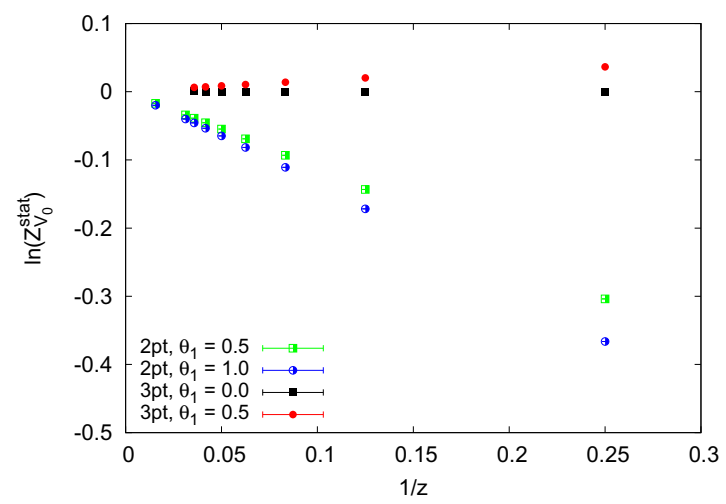

Figure 4: Tree-level continuum results for the renormalization constant of the temporal component of the vector current, $\ln Z_{V_{0}}$, in the static approximation. We compare its definition via two-point correlation functions only (2pt) with our standard definition to fix this HQET parameter, which uses three-point functions (3pt).

From figures 2-4 one can conclude that $\boldsymbol{\theta}_{1}=0$ or small is a good choice for observables depending on a single angle (such as $\ln Z_{\overrightarrow{\mathrm{V}}}$ ), whereas for the parameters via observables depending on two angles (such as $c_{\mathrm{V}_{k, 1}}$ ) the $\boldsymbol{\theta}$-dependence is rather moderate. The parameters depending on three angles (such as $c_{\mathrm{V}_{k, 3}}$ ) show a stronger influence by the $\boldsymbol{\theta}$-choices and sizable effects of higher-order corrections in $1 / z$, which often do not decrease substantially (or not at all) in the "EX" case; thus, these effects appear to be a genuine prop- erty of them. Figure 4 also compares two alternative ways to determine $\ln Z_{\mathrm{V}_{0}}^{\text {stat }}$, either based on two- or threepoint functions $[8,18]$. Owing to the visibly flatter $1 / z$ dependence, the latter is obviously to be preferred, and $\ln Z_{\mathrm{V}_{0}}^{\mathrm{HQET}}$ and $\ln Z_{\overrightarrow{\mathrm{A}}}^{\mathrm{HQET}}$ behave similarly. For more details we refer again to [8]. The matching strategy of [8] is also studied at one-loop order in perturbation theory, see [19] for a preliminary report of results, which support the findings of the tree-level study.

Let us finally come back to the higher-order corrections of $\omega_{\text {kin }}$, which were found to be large compared to all others. They propagate to a large extent into higherorder corrections of the HQET parameters of $A_{0}$ and $V_{k}$, which are the current components required for the calculation of the $\mathrm{B}_{(\mathrm{s})}$-meson decay constant and form factors of semi-leptonic B-meson decays to light mesons. However, as also discussed in [8], it is possible to reduce these higher-order effects in $1 / z$ by removing them completely at tree-level via a straightforward modification of the matching observables. This will be implemented together with the non-perturbative matching computation. Furthermore, there is the option to reduce the higher-order effects in $\omega_{\text {kin }}$ by a generalized set of $\Phi_{i}$, with less zeros in $\varphi$. While this option may reduce the impact of $1 / m_{\mathrm{h}}^{2}$-terms further, we emphasize once more that the proposal of [8] does have $\mathrm{O}(1)$ coefficients as expected generically.

\section{4. $B \rightarrow$ K Form Factor}

In this section, we summarize the exploratory work of the ALPHA Collaboration to determine the form factors for $\mathrm{B}_{\mathrm{s}} \rightarrow \mathrm{K} \ell v$ decays non-perturbatively on the lattice with $N_{\mathrm{f}}=2$ sea quarks [20,21, 22]. For this decay no experimental data is available yet. However, the presence of a heavier spectator s-quark renders the lattice computations technically simpler than for $\mathrm{B} \rightarrow \pi \ell v$. Thus, it offers a good basis to gain solid control on the systematic errors.

The decay amplitude for $\mathrm{B}_{\mathrm{s}} \rightarrow \mathrm{K} \ell v$ is proportional to $\left|V_{\mathrm{ub}}\right|$ times the hadronic matrix element parameterized as

$$
\begin{aligned}
& \left\langle\mathrm{K}\left(p_{\mathrm{K}}\right)\left|V^{\mu}\right| \mathrm{B}_{\mathrm{s}}\left(p_{\mathrm{B}_{\mathrm{s}}}\right)\right\rangle= \\
& =f_{+}\left(q^{2}\right)\left[p_{\mathrm{B}_{\mathrm{s}}}^{\mu}+p_{\mathrm{K}}^{\mu}-\frac{m_{\mathrm{B}_{\mathrm{s}}}^{2}-m_{\mathrm{K}}^{2}}{q^{2}} q^{\mu}\right] \\
& \quad+f_{0}\left(q^{2}\right) \frac{m_{\mathrm{B}_{\mathrm{s}}}^{2}-m_{\mathrm{K}}^{2}}{q^{2}} q^{\mu},
\end{aligned}
$$

where the two form factors $f_{0}\left(q^{2}\right)$ and $f_{+}\left(q^{2}\right)$ depend on $q^{2}=\left(p_{\mathrm{B}_{\mathrm{s}}}-p_{\mathrm{K}}\right)^{2}$, the invariant mass of the lepton 


\begin{tabular}{ccccccc}
\hline id & $T / a \times(L / a)^{3}$ & $a[\mathrm{fm}]$ & $m_{\pi}[\mathrm{MeV}]$ & $m_{\pi} L$ & \# meas. & \# target \\
\hline A5 & $64 \times 32^{3}$ & $0.0749(8)$ & 330 & 4.0 & 500 & 500 \\
F6 & $96 \times 48^{3}$ & $0.0652(6)$ & 310 & 5.0 & 254 & 500 \\
N6 & $96 \times 48^{3}$ & $0.0483(4)$ & 340 & 4.0 & 220 & 500 \\
\hline
\end{tabular}

Table 1: List of ensembles used in [22].

pair. When neglecting the lepton masses, only $f_{+}\left(q^{2}\right)$ contributes to the decay rate.

In order to keep the value of $q^{2}$ fixed in the continuum extrapolation of the form factors, we employ flavour twisted boundary conditions, eq. (15), for the s-quark. By choosing the vector of twist angles, $\boldsymbol{\theta}$, of the s-quark, one can thus freely tune the momentum of the kaon. To remain in the rest frame of the $\mathrm{B}_{\mathrm{s}}$-meson, the heavy quark is twisted by the same angle.

We perform our measurements on gauge field ensembles generated with $N_{\mathrm{f}}=2$ dynamical sea quarks within the CLS effort. They were simulated with nonperturbatively $\mathrm{O}(a)$ improved Wilson fermions and the scale is set via $f_{\mathrm{K}}$ [23], i.e., physical units are derived from $f_{\mathrm{K}}=155 \mathrm{MeV}$. All ensembles have $m_{\pi} L \gtrsim 4$ where finite-volume effects can be neglected. In this work, we use the three CLS ensembles A5, F6 and N6, described in [23]. Our error estimates take into account correlations and autocorrelations [24].

In the exploratory study presented here, we restrict our analysis to the static approximation because the non-perturbative determination of the full set of HQET parameters is still in progress. Thus we set $\omega_{\text {kin }}=$ $\omega_{\text {spin }}=c_{\mathrm{V}}=0$. For the renormalization constants to be used at static order in eqs. (11) and (16), we follow the lines of $[13,25]$ and write

$$
\begin{aligned}
& Z_{\mathrm{V}_{0}}^{\mathrm{HQET}}=C_{\mathrm{PS}}\left(M_{\mathrm{h}} / \Lambda_{\overline{\mathrm{MS}}}\right) Z_{\mathrm{A}, \mathrm{RGI}}^{\mathrm{stat}}\left(g_{0}\right) Z_{\mathrm{V} / \mathrm{A}}^{\mathrm{stat}}\left(g_{0}\right), \\
& Z_{\mathrm{V}_{\mathrm{k}}}^{\mathrm{HQET}}=C_{\mathrm{V}}\left(M_{\mathrm{h}} / \Lambda_{\overline{\mathrm{MS}}}\right) Z_{\mathrm{A}, \mathrm{RGI}}^{\mathrm{stat}}\left(g_{0}\right) .
\end{aligned}
$$

The matching factors, $C_{\mathrm{PS}}$ and $C_{\mathrm{V}}$, are known at three loops in perturbation theory [26], and $Z_{\mathrm{A}, \mathrm{RGI}}^{\mathrm{stat}}$ is known non-perturbatively [27]. The truncation of the theory at static order is expected to be a 10-20\% effect.

For an analysis in the static approximation, we only need to consider the two- and three-point correlation functions (while in the same measurement runs we also determine the various correlation functions that are needed for the extraction of the form factors in HQET at order $1 / m_{\mathrm{h}}$ )

$$
C^{\mathrm{K}}\left(x^{0}-y^{0} ; \mathbf{p}\right)=\sum_{\mathbf{x}, \mathbf{y}} \mathrm{e}^{-\mathrm{i} \mathbf{p} \cdot(\mathbf{x}-\mathbf{y})}\left\langle P^{\mathrm{su}}(x) P^{\mathrm{us}}(y)\right\rangle,
$$

$$
\begin{aligned}
C_{i j}^{\mathrm{B}}\left(x^{0}-y^{0} ; \mathbf{0}\right)= & \sum_{\mathbf{x}, \mathbf{y}}\left\langle P_{i}^{\mathrm{sb}}(x) P_{j}^{\mathrm{bs}}(y)\right\rangle, \\
C_{\mu, j}^{3}\left(t_{\mathrm{K}}, t_{\mathrm{B}} ; \mathbf{p}\right)= & \sum_{\mathbf{x}_{\mathrm{K}}, \mathbf{x}_{V}, \mathbf{x}_{\mathrm{B}}} \mathrm{e}^{-\mathrm{i} \mathbf{p} \cdot\left(\mathbf{x}_{\mathrm{K}}-\mathbf{x}_{V}\right)} \\
& \times\left\langle P^{\mathrm{su}}\left(x_{\mathrm{K}}\right) \mathrm{V}_{\mu}^{\mathrm{stat}}\left(x_{V}\right) P_{j}^{\mathrm{bs}}\left(x_{\mathrm{B}}\right)\right\rangle .
\end{aligned}
$$

The $P_{i}^{\mathrm{q}_{1} \mathrm{q}_{2}}(x)$ are interpolating fields, such as $\bar{\psi}_{\mathrm{q}_{1}}(x) \gamma_{5} \psi_{\mathrm{q}_{2}}(x)$, for the mesons. Different levels of Gaussian smearing [28] of the s-quark in the heavylight meson, i.e., different trial wave functions, are indicated by the subscripts $i$ or $j$. For any suitable smearing $i$, the ratio

$$
f_{\mu, i}^{\mathrm{ratio}}\left(t_{\mathrm{B}}, t_{\mathrm{K}} ; q^{2}\right) \equiv \frac{C_{\mu, i}^{3}\left(t_{\mathrm{K}}, t_{\mathrm{B}}\right)}{\sqrt{C^{\mathrm{K}}\left(t_{\mathrm{K}}\right) C_{i i}^{\mathrm{B}}\left(t_{\mathrm{B}}\right)}} \mathrm{e}^{\frac{E_{\mathrm{K}} t_{\mathrm{K}}}{2}} \mathrm{e}^{\frac{E_{\mathrm{B}} t_{\mathrm{B}}}{2}}
$$

will give the desired matrix element in the limit of large Euclidean times, $t_{\mathrm{B}} \equiv x_{\mathrm{B}}^{0}-x_{V}^{0}$ and $t_{\mathrm{K}} \equiv x_{V}^{0}-x_{\mathrm{K}}^{0}$ :

$$
\left\langle\mathrm{K}\left(p_{\mathrm{K}}^{\theta}\right)\left|\mathrm{V}_{\mu}\right| \mathrm{B}_{\mathrm{s}}(0)\right\rangle=\lim _{t_{\mathrm{B}}, t_{\mathrm{K}} \rightarrow \infty} f_{\mu, i}^{\mathrm{ratio}}\left(t_{\mathrm{B}}, t_{\mathrm{K}} ; q^{2}\right) .
$$

Alternatively, we can parameterize the $t_{\mathrm{B}^{-}}$and $t_{\mathrm{K}^{-}}$ dependence of the correlation functions as

$$
\begin{aligned}
C^{\mathrm{K}}\left(t_{\mathrm{K}}\right) & =\sum_{m}\left(\kappa^{(m)}\right)^{2} \mathrm{e}^{-E_{\mathrm{K}}^{(m)} t_{\mathrm{K}}}, \\
C_{i j}^{\mathrm{B}}\left(t_{\mathrm{B}}\right) & =\sum_{n} \beta_{i}^{(n)} \beta_{j}^{(n)} \mathrm{e}^{-E_{\mathrm{B}}^{(n)} t_{\mathrm{B}}}, \\
C_{\mu, i}^{3}\left(t_{\mathrm{B}}, t_{\mathrm{K}}\right) & =\sum_{m, n} \kappa^{(m)} \varphi_{\mu}^{(m, n)} \beta_{i}^{(n)} \mathrm{e}^{-E_{\mathrm{B}}^{(n)} t_{\mathrm{B}}} \mathrm{e}^{-E_{\mathrm{K}}^{(m)} t_{\mathrm{K}}},
\end{aligned}
$$

and determine $\left\{\kappa^{(m)}, E_{\mathrm{K}}^{(m)}\right\}$ from a fit to $C^{\mathrm{K}}$, and $\left\{\beta_{i}^{(n)}, \varphi_{\mu}^{(n, m)}, E_{\mathrm{B}}^{(n)}\right\}$ from a combined fit to $C_{\mu, i}^{3}$ and $C_{i j}^{\mathrm{B}}$. The matrix element of eq. (26) is then given by the fit parameter $\varphi_{\mu}^{(1,1)}$. We include the first excited $\mathrm{B}_{\mathrm{s}}$-state, but only the kaon ground state (i.e., we take $m=1$ and $n=1,2)$.

The ratio $f_{\mu}^{\text {ratio }}$ of eq. (25) is shown as a function of $t_{\mathrm{B}}$ at fixed $t_{\mathrm{K}}=20$ in figure 5 . For comparison, the value of $\varphi_{\mu}^{(1,1)}$ resulting from the fit is indicated as a red band.

In the rest frame of the $\mathrm{B}_{\mathrm{s}}$-meson, the matrix elements have the form

$$
\begin{aligned}
\left\langle\mathrm{K}\left|\mathrm{V}_{0}\right| \mathrm{B}_{\mathrm{s}}\right\rangle & =\sqrt{2 m_{\mathrm{B}_{\mathrm{s}}}} f_{\|}\left(q^{2}\right), \\
\left\langle\mathrm{K}\left|\mathrm{V}_{i}\right| \mathrm{B}_{\mathrm{s}}\right\rangle & =\sqrt{2 m_{\mathrm{B}_{\mathrm{s}}}} p_{\mathrm{K}}^{i} f_{\perp}\left(q^{2}\right),
\end{aligned}
$$




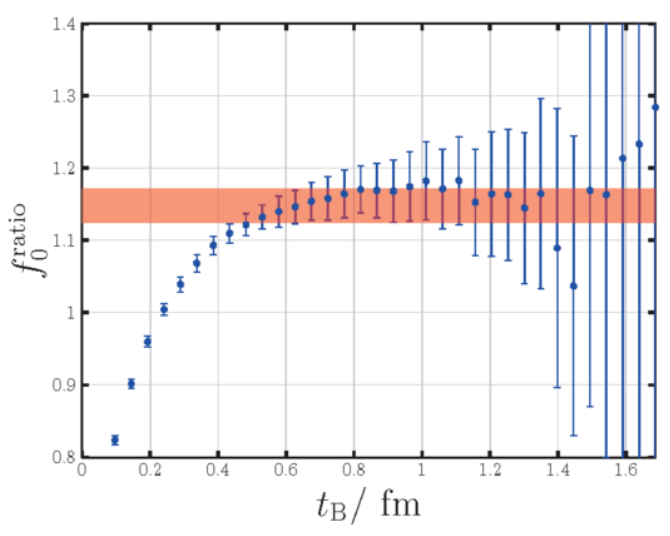

Figure 5: The ratio $f_{\mu}^{\text {ratio }}$ (points) and the fit result $\varphi_{\mu}^{(1,1)}$ (band) for lattice N6, $\mu=0$ and fixed $t_{\mathrm{K}}=20$.

where the form factors $\left(f_{\|}, f_{\perp}\right)$ are related to $\left(f_{+}, f_{0}\right)$ by

$$
\begin{aligned}
f_{+}= & \frac{1}{\sqrt{2 m_{\mathrm{B}_{\mathrm{s}}}}} f_{\|}+\frac{1}{\sqrt{2 m_{\mathrm{B}_{\mathrm{s}}}}}\left(m_{\mathrm{B}_{\mathrm{s}}}-E_{\mathrm{K}}\right) f_{\perp}, \\
f_{0}= & \frac{\sqrt{2 m_{\mathrm{B}_{\mathrm{s}}}}}{m_{\mathrm{B}_{\mathrm{s}}}^{2}-m_{\mathrm{K}}^{2}}\left[\left(m_{\mathrm{B}_{\mathrm{s}}}-E_{\mathrm{K}}\right) f_{\|}\right. \\
& \left.+\left(E_{\mathrm{K}}^{2}-m_{\mathrm{K}}^{2}\right) f_{\perp}\right] .
\end{aligned}
$$

The form factor $f_{+}$extracted from the fitted $\varphi_{\mu}^{(1,1)}$ at different lattice spacings is shown in figure 6. When computing $f_{+}$in the static approximation of HQET, we are free to keep or drop terms of order $1 / m_{\mathrm{h}}$ in eq. (27). To illustrate the corresponding $\mathrm{O}\left(1 / m_{\mathrm{h}}\right)$ ambiguity, we show in figure 6 (and 7) two sets of data points: the upper one corresponds to using all terms in eq. (27), the lower one to dropping the term proportional to $f_{\|}$. This ambiguity will disappear, once all $\mathrm{O}\left(1 / m_{\mathrm{h}}\right)$ terms of HQET are included. For both sets we show a constant continuum extrapolation and one linear in $a^{2}$. The latter has by far the larger error, and within this error it is consistent with the result of the constant extrapolation.

Figure 7 shows a comparison of our results from the linear continuum extrapolation of $f_{+}\left(q^{2}\right)$ with recent results of HPQCD [29] (at their smallest $a=0.09 \mathrm{fm}$ and $\left.m_{\pi}=320 \mathrm{MeV}\right)$.

\section{Conclusions}

With the matching conditions between HQET at $\mathrm{O}\left(1 / m_{\mathrm{h}}\right)$ and QCD reviewed here, it has been demonstrated that the solution of the extended matching problem, including all components of the axial and vector currents, is feasible. Our lattice formulation basically

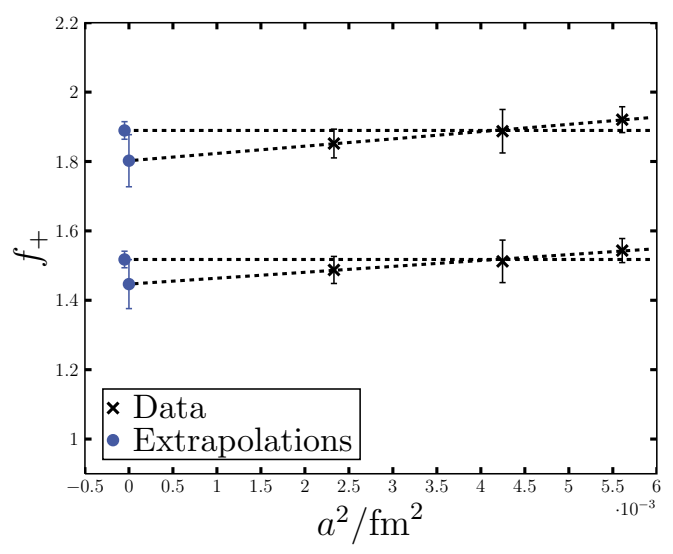

Figure 6: Continuum extrapolation of our data for $f_{+}$at $q^{2}=$ $21.23 \mathrm{GeV}^{2}$

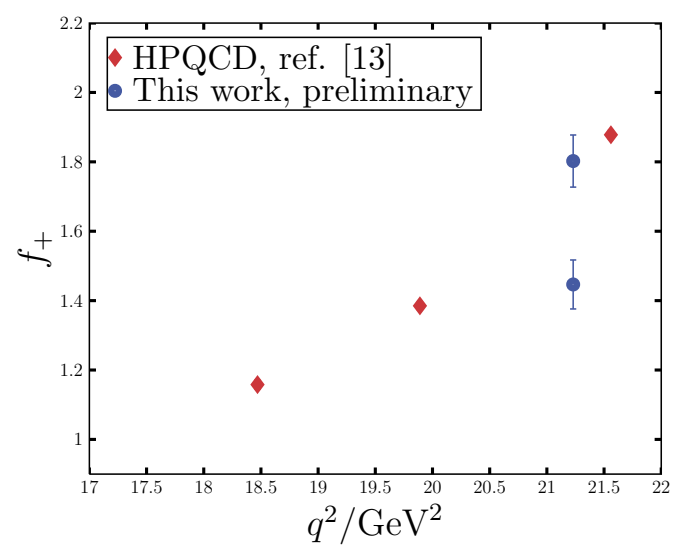

Figure 7: Comparison of lattice QCD results at various values of $q^{2}$.

amounts to solving a linear system of matching equations with the 19 HQET parameters as unknowns. The matrix associated with this system has a simple block structure. The realization of this matching strategy employs observables which are constructed from correlation functions in a finite volume with Schrödinger functional boundary conditions, and which would correspond to mesonic correlation functions in large volume. We exploit particular kinematic settings that are well suited for a straightforward implementation on the non-perturbative level by numerical simulations.

Although our matching strategy (entirely expressed through renormalized quantities in QCD) is not necessarily restricted to Wilson fermions, we will use these in the future non-perturbative computation. According to our past experience with Wilson fermions $[6,7]$, very fine lattice spacings and good numerical precision could be achieved. Moreover, with our tree-level study of the $1 / z$-dependence of the HQET parameters we have il- 
lustrated that the linear behaviour already sets in for $z \geq 8$ in most cases, with slopes of $\mathrm{O}(1)$. In fact, this finding is very reassuring, as it implies that for the non-perturbative framework followed by our collaboration [7], with a matching volume of extent $L \approx 0.5 \mathrm{fm}$ and dimensionless heavy quark masses around $z=13$ at the b-quark mass scale, higher-order corrections beyond the already included $\mathrm{O}\left(1 / m_{\mathrm{h}}\right)$ ones are suppressed by a factor of about 10 .

The non-perturbative solution of the HQET matching problem is a crucial step towards a precision computation of phenomenologically relevant hadronic matrix elements in B-physics. We presented here the current status of our effort to compute form factors for semileptonic B-decays using HQET on the lattice. In particular, we considered the decay $\mathrm{B}_{\mathrm{s}} \rightarrow \mathrm{K} \ell v$ and investigated two different methods to extract the form factor $f_{+}\left(q^{2}\right)$ at a fixed value of $q^{2}=21.23 \mathrm{GeV}^{2}$ from the plateau value of a suitable ratio of correlators or from a simultaneous fit to the functional form of the correlators.

The authors of [22] performed a continuum extrapolation of the lattice data for $f_{+}\left(q^{2}\right)$ and found very small $\mathrm{O}\left(a^{2}\right)$ effects. While these preliminary results were still computed in the static approximation and an extrapolation to the physical pion mass has yet to be performed, the preliminary value of $f_{+}$is in agreement with the results from other collaborations at the same quark mass.

There is thus no sign of reduction of the difference of $V_{\mathrm{ub}}$ extracted from semi-leptonic and inclusive/leptonic decays, but at the moment there are 10-20\% uncertainties due to neglecting the $1 / m_{\mathrm{h}}$-terms. These terms have to be included for a firm conclusion. Once the HQET parameters are known non-perturbatively, they will all be included in the analysis. Moreover, we plan to extend the computation to $\mathrm{B} \rightarrow \pi \ell v$ decays, several values of $q^{2}$, and $N_{\mathrm{f}}=2+1$ flavours of sea quarks.

Acknowledgements. We thank Felix Bahr, Fabio Bernardoni, John Bulava, Michael Donnellan, Samantha Dooling, Dirk Hesse, Anosh Joseph and Piotr Korcyl for a pleasant collaboration and many useful discussions. In particular we received many valuable comments by Piotr Korcyl on the topics of this review.

We are grateful for the support of the Deutsche Forschungsgemeinschaft (DFG) in the SFB/TR 09 "Computational Particle Physics" and we have profited very much from the scientific exchange in the SFB. This work was partially supported by the Spanish Minister of Education and Science, project RyC-2011-08557
(M. D. M.), and by the grant HE $4517 / 3-1$ of the DFG (J. H.).

We gratefully acknowledge the Gauss Centre for $\mathrm{Su}$ percomputing (GCS) for providing computing time through the John von Neumann Institute for Computing (NIC) on the GCS share of the supercomputer JUQUEEN at Jülich Supercomputing Centre (JSC). GCS is the alliance of the three national supercomputing centres HLRS (Universität Stuttgart), JSC (Forschungszentrum Jülich), and LRZ (Bayerische Akademie der Wissenschaften), funded by the German Federal Ministry of Education and Research (BMBF) and the German State Ministries for Research of Baden-Württemberg (MWK), Bayern (StMWFK) and Nordrhein-Westfalen (MIWF). We acknowledge PRACE for awarding us access to resource JUQUEEN in Germany at Jülich and to resource SuperMUC based in Germany at the LRZ, Munich. We also thank the LRZ for a CPU time grant on SuperMUC, project pr85ju, and DESY for access to the PAX cluster in Zeuthen.

\section{References}

[1] R. Sommer, Non-perturbative Heavy Quark Effective Theory: Introduction and Status, SFB TR9 - Computational Particle Physics, SFB/CPP-14-101.

[2] K. Olive, et al., Review of Particle Physics, Chin.Phys. C38 (2014) 090001. doi:10.1088/1674-1137/38/9/090001.

[3] S. Aoki, Y. Aoki, C. Bernard, T. Blum, G. Colangelo, et al., Review of lattice results concerning low-energy particle physics, Eur.Phys.J. C74 (9) (2014) 2890. arXiv:1310.8555, doi:10.1140/epjc/s10052-014-2890-7.

[4] C. Bourrely, I. Caprini, L. Lellouch, Model-independent description of $\mathrm{B} \rightarrow \pi \ell v$ decays and a determination of $-\mathrm{V}(\mathrm{ub})$ - $\quad$ Phys.Rev. D79 (2009) 013008. arXiv:0807.2722, doi:10.1103/PhysRevD.82.099902, 10.1103/PhysRevD.79.013008.

[5] J. Heitger, R. Sommer, Nonperturbative heavy quark effective theory, JHEP 0402 (2004) 022. arXiv:hep-lat/0310035, doi:10.1088/1126-6708/2004/02/022.

[6] B. Blossier, M. Della Morte, N. Garron, R. Sommer, HQET at order $1 / \mathrm{m}$ : I. Non-perturbative parameters in the quenched approximation, JHEP 1006 (2010) 002. arXiv:1001.4783, doi:10.1007/JHEP06(2010)002.

[7] B. Blossier, M. Della Morte, P. Fritzsch, N. Garron, J. Heitger, R. Sommer, H. Simma, N. Tantalo, Parameters of Heavy Quark Effective Theory from $N_{\mathrm{f}}=2$ lattice QCD, JHEP 1209 (2012) 132. arXiv:1203.6516, doi:10.1007/JHEP09(2012)132.

[8] M. Della Morte, S. Dooling, J. Heitger, D. Hesse, H. Simma, Matching of heavy-light flavor currents between HQET at order 1/m and QCD: I. Strategy and tree-level study, JHEP 1405 (2014) 060. arXiv:1312.1566, doi:10.1007/JHEP05(2014)060.

[9] S. Sint, R. Sommer, The running coupling from the QCD Schrödinger functional: A one loop analysis, Nucl. Phys. B465 (1996) 71-98. arXiv:hep-lat/9508012.

[10] M. Lüscher, S. Sint, R. Sommer, P. Weisz, Chiral symmetry and O(a) improvement in lattice QCD, Nucl.Phys. B478 (1996) 365400. arXiv:hep-lat/9605038, doi:10.1016/0550-3213(96)00378 1. 
[11] P. F. Bedaque, Aharonov-Bohm effect and nucleon nucleon phase shifts on the lattice, Phys.Lett. B593 (2004) 82-88. arXiv:nucl-th/0402051, doi:10.1016/j.physletb.2004.04.045.

[12] C. Sachrajda, G. Villadoro, Twisted boundary conditions in lattice simulations, Phys.Lett. B609 (2005) 73. arXiv:heplat/0411033, doi:10.1016/j.physletb.2005.01.033.

[13] R. Sommer, Introduction to Non-perturbative Heavy Quark Effective Theory, published in Les Houches Summer School in Theoretical Physics, Modern perspectives in lattice QCD, Les Houches, France, 3 - 28 August 2009, Oxford Univ. Pr. (2010) 517arXiv:1008.0710.

[14] M. Lüscher, R. Narayanan, P. Weisz, U. Wolff, The Schrodinger functional: A Renormalizable probe for non-Abelian gauge theories, Nucl.Phys. B384 (1992) 168-228. arXiv:heplat/9207009, doi:10.1016/0550-3213(92)90466-O.

[15] S. Sint, On the Schrodinger functional in QCD, Nucl.Phys. B421 (1994) 135-158. arXiv:hep-lat/9312079, doi:10.1016/0550-3213(94)90228-3.

[16] S. Sint, One loop renormalization of the QCD Schrodinger functional, Nucl.Phys. B451 (1995) 416-444. arXiv:heplat/9504005, doi:10.1016/0550-3213(95)00352-S.

[17] S. Sint, P. Weisz, Further results on $\mathrm{O}(a)$ improved lattice QCD to one-loop order of perturbation theory, Nucl. Phys. B502 (1997) 251. arXiv:hep-lat/9704001.

[18] D. Hesse, R. Sommer, A one-loop study of matching conditions for static-light flavor currents, JHEP 1302 (2013) 115. arXiv:1211.0866, doi:10.1007/JHEP02(2013)115.

[19] P. Korcyl, Fixing the parameters of Lattice HQET including 1/ $m_{\mathrm{B}}$ terms, PoS Beauty2013 (2013) 071. arXiv:1307.5080.

[20] F. Bahr, et al., $\left|V_{u b}\right|$ determination in lattice QCD, PoS ICHEP2012 (2013) 424. arXiv:1211.6327.

[21] F. Bahr, F. Bernardoni, A. Ramos, H. Simma, R. Sommer, et al., $\mathrm{B} \rightarrow \pi$ form factor with 2 flavours of $O(a)$ improved Wilson quarks, PoS LATTICE2012 (2012) 110. arXiv:1210.3478.

[22] F. Bahr, F. Bernardoni, J. Bulava, A. Joseph, A. Ramos, et al., Form factors for $\mathrm{B}_{\mathrm{s}} \rightarrow \mathrm{K} \ell v$ decays in Lattice QCD. arXiv: 1411.3916.

[23] P. Fritzsch, F. Knechtli, B. Leder, M. Marinkovic, S. Schaefer, et al., The strange quark mass and Lambda parameter of two flavor QCD, Nucl.Phys. B865 (2012) 397-429. arXiv:1205.5380, doi:10.1016/j.nuclphysb.2012.07.026.

[24] S. Schaefer, R. Sommer, F. Virotta, Critical slowing down and error analysis in lattice QCD simulations, Nucl.Phys. B845 (2011) 93-119. arXiv:1009.5228, doi:10.1016/j.nuclphysb.2010.11.020.

[25] J. Heitger, A. Jüttner, R. Sommer, J. Wennekers, Nonperturbative tests of heavy quark effective theory, JHEP 0411 (2004) 048. arXiv:hep-ph/0407227, doi:10.1088/11266708/2004/11/048

[26] K. Chetyrkin, A. Grozin, Three loop anomalous dimension of the heavy light quark current in HQET, Nucl.Phys. B666 (2003) 289-302. arXiv:hep-ph/0303113, doi:10.1016/S05503213(03)00490-5.

[27] M. Della Morte, P. Fritzsch, J. Heitger, Non-perturbative renormalization of the static axial current in two-flavour QCD, JHEP 0702 (2007) 079. arXiv:hep-lat/0611036, doi:10.1088/11266708/2007/02/079.

[28] Güsken, S. and Löw, U. and Mutter, K.H. and Sommer, R. and Patel, A. and others, Nonsinglet Axial Vector Couplings of the Baryon Octet in Lattice QCD, Phys.Lett. B227 (1989) 266. doi:10.1016/S0370-2693(89)80034-6.

[29] C. Bouchard, G. P. Lepage, C. Monahan, H. Na, J. Shigemitsu, $B_{s} \rightarrow K \ell v$ form factors from lattice QCD, Phys.Rev. D90 (2014) 054506. arXiv:1406.2279, doi:10.1103/PhysRevD.90.054506. 